\title{
Association between marriage and outcomes in patients with acute ischemic stroke
}

\author{
Qi Liu' ${ }^{1}$ Xianwei Wang ${ }^{2,3,4,5} \cdot$ Yilong Wang ${ }^{2,3,4,5} \cdot$ Chunxue Wang ${ }^{2,3,4,5} \cdot$ Xingquan Zhao $^{2,3,4,5} \cdot$ Liping Liu $^{2,3,4,5}$. \\ Zixiao $\mathrm{Li}^{2,3,4,5} \cdot$ Xia Meng ${ }^{2,3,4,5} \cdot \mathrm{Li} \mathrm{GuO}^{1} \cdot$ Yongjun Wang ${ }^{2,3,4,5}$
}

Received: 27 December 2017 / Revised: 8 February 2018 / Accepted: 9 February 2018 / Published online: 20 February 2018

(C) The Author(s) 2018. This article is an open access publication

\begin{abstract}
Backgrounds The previous studies on the association between marital status and stroke outcomes were rare. Furthermore, the existing studies mostly focused on the protective effect of marriage on survival. We conducted the study to evaluate the association between marital status and adverse stroke outcomes in patients with AIS based on China national stroke registry. Methods This was a multicenter, prospective cohort study of patients with AIS. Patients were classified into two groups based on marital status at admission: married and unmarried. The primary outcomes included all-cause mortality, stroke recurrence, combined endpoint, and stroke disability. Stroke disability was defined as modified Rankin Scale of 2-6.

Results Of 12,118 patients, 1220 were unmarried and 10,898 married. Unmarried patients had higher proportion of 1-year post-stroke events than married patients did. As compared with being unmarried, the adjusted odds ratios with $95 \%$ confidence interval of being married for outcomes were as follows: $0.70(0.58-0.84)$ for all-cause mortality, $0.78(0.66-0.91)$ for stroke recurrence, $0.77(0.66-0.90)$ for combined endpoint, and $0.75(0.65-0.88)$ for stroke disability. Interactions between marital status and education were significant for all outcomes except for stroke disability.

Conclusions Marital status was associated with all adverse stroke outcomes in patients with acute ischemic stroke, especially in those with middle-school education.
\end{abstract}

Keywords Acute ischemic stroke $\cdot$ Marital status $\cdot$ All-cause death $\cdot$ Stroke recurrence $\cdot$ Stroke disability

Qi Liu and Xianwei Wang have contributed equally to the manuscript.

Electronic supplementary material The online version of this article (https://doi.org/10.1007/s00415-018-8793-z) contains supplementary material, which is available to authorized users.

Li Guo

guoli6hb@163.com

$\triangle$ Yongjun Wang

yongjunwang1962@gmail.com

1 Department of Neurology, The Second Hospital of Hebei Medical University, No.215 Hepingxilu, Xinhua District, Shijiazhuang 050000, Hebei, China

2 Department of Neurology, Beijing Tiantan Hospital, Capital Medical University, No.6 Tiantanxili, Dongcheng District, Beijing 100050, China

\section{Introduction}

Stroke is the leading cause of death and adult disability in the worldwide [1,2]. China has more than 2.5 million new stroke cases each year, and ischemic stroke accounts for $43-79 \%$ of all strokes [3]. Controlling risk factors would contribute to stroke prevention and improving survival outcomes. Studies demonstrated that marriage was negatively

3 China National Clinical Research Center for Neurological Diseases, Beijing 100050, China

4 Center of Stroke, Beijing Institute for Brain Disorders, Beijing 100050, China

5 Beijing Key Laboratory of Translational Medicine for Cerebrovascular Disease, Beijing 100050, China 
associated with adverse cardiovascular events [4] and was an independent predictor of survival [5, 6].

Stroke patients might need more social or family supports due to stroke disability. Existing literatures show that unmarried patients are more likely to die following a stroke than married patients [7, 8]. However, there is little data on impact of marriage on recurrent stroke and poor functional outcome, especially among Asian population. We hypothesized that marriage would a protective effect on adverse outcomes in patients with acute ischemic stroke (AIS). Thus, the study aimed to evaluate the association between marital status and stroke outcomes including all-cause mortality, stroke recurrence, and stroke disability based on the China National Stroke Registry (CNSR).

\section{Methods}

\section{Study population}

All study subjects were from a nationwide CNSR which was designed to evaluate the quality of care for hospitalized stroke patients and measure the clinical and functional outcomes at 1 year after disease onset. Details about study rational, design, and results have been published previously [9]. For the present study, subjects were patients who met the following criteria: (1) older than 18; (2) diagnosis of AIS within 14 days after the onset of symptoms, and which are confirmed by brain computed tomography or magnetic resonance imaging; and (3) written informed consent obtained from patients or their legally authorized representatives. From September 2007 to August 2008, 12,415 consecutive patients were included, 233 (1.9\%) patients were excluded for missing 1-year follow-up, and $64(0.5 \%)$ were excluded for missing marital status data. Finally, 12,118 (97.6\%) patients were analyzed into the current study. (Supplemental figure 1) The study was approved by the central institutional review board at Beijing Tiantan Hospital.

\section{Data collection}

Data collection was completed by trained research coordinators according to a standard protocol. The baseline data extracted from the medical records included patient demographics, socioeconomic status, medical conditions, NIHSS (National Institutes of Health Stroke Scale), and other vascular risk factors. The medical conditions included history of stroke, hypertension, diabetes, dyslipidaemia, atrial fibrillation, coronary heart disease, and pneumonia. Other vascular risk factors included current or previous smoking and moderate or heavy alcohol consumption ( $\geq 2$ standardized alcohol drinks per day) and body mass index (BMI). Socioeconomic status (SES) included educational level, occupational class, and personal income level [10]. Occupational class was classified based on the main job types at admission. Personal income refers average family income per capita per month (e.g., the family's actual income per month is divided by the number of family members).

\section{Marital status grouping}

Marital status included married, never married, remarried, widowed, and divorced. In the current study, all subjects were divided into two categories: married and unmarried. Considering the small sample of remarried $(0.21 \%)$, never married $(1.13 \%)$, and divorced $(0.58 \%)$, being married included married and remarried while being unmarried included widowed, divorced, and never married [5].

\section{Outcome assessment}

One-year telephone follow-up was conducted by trained research personnel who was blinded to patient's baseline characteristics. Outcomes included all-cause mortality, stroke recurrence, combined endpoint (death and stroke recurrence), and stroke disability. Stroke recurrence contained ischemic stroke, intracranial hemorrhage, and subarachnoid hemorrhage. During the follow-up periods, stroke recurrence associated with rehospitalization was sourced to the attended hospitals to ensure a reliable diagnosis. In the case of a suspected recurrent cerebrovascular event without hospitalization, judgement was made by the research coordinators together with the principal investigator [9]. Stroke disability was defined as modified Rankin Scale of 2-6 [mRS, score ranges from 0 (no symptoms) to 6 (death)].

\section{Statistical analysis}

We compared baseline and clinical characteristics of patients grouped by marital status by $t$ test and $\chi^{2}$ or Fisher's exact test. Continuous variables were expressed as median (interquartile range, IQR), whereas categorical data were presented as proportions.

Multivariable logistic regression model was performed to assess the association between marital status and stroke outcomes with the unmarried group as the reference. The multivariable analysis adjusted the following covariates which were thought to be associated with adverse outcomes: age, sex, region, types of health insurance, history of stroke, hypertension, diabetes, dyslipidaemia, atrial fibrillation, coronary heart disease, pneumonia, current or previous smoking, moderate or heavy alcohol, body mass index (BMI) at admission, baseline NIHSS, living status, and SES. Odds ratios (ORs) with 95\% confidence intervals (CIs) were calculated. 
We also performed stratification analyses by age $(\leq 65$ and $>65$ years), education (elementary school or below, middle school, and high school or above), gender (male and female), and region (eastern, central and western). To examine effect modification by age, education, gender, and region, we used a post-estimation Wald test in multivariable-adjusted logistic model to get an omnibus $P$ value for interaction between marital status categories and variables of interest.

A two-sided $P$ value $<0.05$ was set as the level for statistical significance. All analyses were performed with SAS software version 9.3 (SAS Institute Inc, Cary, NC, USA).

\section{Results}

\section{Study population and characteristics}

Of the final 12,118 patients in this study, $1220(10.1 \%)$ were unmarried and 10,898 (89.9\%) married (Supplementary Fig. 1). The median age was 67 years. As compared with married, unmarried patients were older, more likely to be female, to have lower BMI at baseline, to live alone, and to have elementary or below education, to be non-working status (retired or no job). In addition, unmarried patients were more likely to have severe stroke, to have a history of coronary heart disease, atrial fibrillation, and pneumonia. Conversely, they were less likely to be smoker and heavy drinker, to live in eastern region, and to have diabetes mellitus and hyperlipidemia than married patients did. There was no significant difference in personal income, history of stoke, and hypertension between the two groups (Table 1).

\section{One-year incidence of stroke outcomes}

Unmarried patients had higher proportion of 1-year poststroke events than married patients: 25.3 versus $12.3 \%$ for all-cause mortality $(P<0.01), 28.5$ versus $18.2 \%$ for stroke recurrence $(P<0.01), 33.4$ versus $21.4 \%$ for combined endpoint $(P<0.01)$, and 61.7 versus $42.6 \%$ for stroke disability $(P<0.01)$, respectively (Fig. 1$)$.

\section{Association between marital status and stroke outcomes}

ORs with 95\% CI of marital status for stroke outcomes were reported in Fig. 2. Univariate and multivariate analyses showed that marital status was independently associated with stroke outcomes. As compared with unmarried, the adjusted ORs of married patients were 0.70 (95\% CI $0.58-0.84$ ) for all-cause mortality, 0.78 (95\% CI 0.66-0.91) for stroke recurrence, 0.77 (95\% CI 0.66-0.90) for combined end point, and 0.75 (95\% CI 0.65-0.88) for stroke disability, respectively.

ORs and 95\% CI of marital status for stroke outcomes in subjects stratified by age, gender, region, and education were shown in Table 2. We found that marriage was independently associated with all stroke outcomes among patients with the middle-school education: 0.43 (95\% CI 0.29-0.65) for all-cause mortality, 0.54 (95\% CI 0.38-0.77) for stroke recurrence, 0.52 (95\% CI 0.37-0.72) for combined endpoint, and 0.60 (95\% CI 0.43-0.83) for stroke disability. In addition, significant interactions between marriage and education for all-cause mortality $(P=0.047)$ and combined endpoint $(P=0.05)$ were also identified, but not for stroke recurrence $(P=0.07)$ and stroke disability $(P=0.35)$. There were no significant interactions between marriage status and age, gender, or region for all stroke outcomes.

In our study, we did not find the association between living arrangements and post-stroke outcomes among unmarried patients (Supplementary Table 1). In addition, multivariate analysis also showed that the association between marital status and stroke outcomes was similar, regardless of whether occupational class and personal income level were the adjustment factors (Supplementary Table 2).

\section{Discussion}

The current study demonstrated that marital status was independently associated with post-stroke outcomes, especially in patients with middle-school education. The 1-year incidences of stroke outcomes after AIS in unmarried patients were approximately 1.5-2.0 times as high as those in married patients.

To our knowledge, the previous studies on the association between marital status and stroke outcomes were rare. Furthermore, the existing studies mostly focused on the protective effect of marriage on survival. Stroke recurrence and disability as severe adverse outcomes were rarely concerned. A recent study published in $J$ Am Heart Ass. found that marital history was significantly associated with survival after stroke. However, this study population was derived from a prospective cohort study of US adults based on patients' reports of stroke according to interviews rather than precise clinical data [7]. Some other studies on marital status and mortality in elderly based on national population included a systematic review and meta-analysis [6]. Our study assessed the association of marital status and stroke outcomes included all-cause mortality, stroke recurrence, combined endpoint, and stroke disability based on a nationwide prospective cohort study of hospitalized stroke patients. However, a recent study showed that the social isolation rather than marital status was associated with all-cause death after stroke [11]. It was a small study with 655 stroke patients and 
Table 1 Baseline characteristics of patients with acute ischemic stroke according to marital status categories

\begin{tabular}{|c|c|c|c|c|}
\hline Characteristics & Overall $(n=12,118)$ & Unmarried $(n=1220)$ & Married $(n=10,898)$ & $P$ value \\
\hline Age (years), median (IQR) & $67(57-75)$ & $77(69-82)$ & $66(56-74)$ & $<0.001$ \\
\hline Female, no. (\%) & $4634(38.2)$ & $749(61.4)$ & $3885(35.6)$ & $<0.001$ \\
\hline BMI at admission $\left(\mathrm{kg} / \mathrm{m}^{2}\right)$, no. $(\%)$ & & & & 0.001 \\
\hline$<25$ & $6643(60.7)$ & $729(65.5)$ & $5914(60.2)$ & \\
\hline $25-30$ & $3713(33.9)$ & $323(29.0)$ & $3390(34.5)$ & \\
\hline$>30$ & $582(5.3)$ & $61(5.5)$ & $521(5.3)$ & \\
\hline Region, no. (\%) & & & & 0.036 \\
\hline Eastern & $7678(63.4)$ & $737(60.4)$ & $6941(63.7)$ & \\
\hline Central & $2525(20.8)$ & $287(23.5)$ & $2238(20.5)$ & \\
\hline Western & $1915(15.8)$ & $196(16.1)$ & $1719(15.8)$ & \\
\hline Ever smoking, no. (\%) & $4826(39.8)$ & $344(28.2)$ & $4482(41.1)$ & $<0.001$ \\
\hline Heavy alcohol, no. (\%) & $1147(9.5)$ & $74(6.1)$ & $1073(9.8)$ & $<0.001$ \\
\hline Types of health insurance, no. (\%) & & & & 0.031 \\
\hline BHIS & 7293 (61.7) & $741(61.9)$ & $6552(61.7)$ & \\
\hline NCMS & $1981(16.8)$ & $170(14.2)$ & $1811(17.1)$ & \\
\hline Self-payment & $2262(19.1)$ & $256(21.4)$ & 2006 (18.9) & \\
\hline Other & $279(2.4)$ & $30(2.5)$ & $249(2.3)$ & \\
\hline \multicolumn{5}{|l|}{ Medical conditions, no. (\%) } \\
\hline History of stroke & $4142(34.2)$ & $435(35.7)$ & $3707(34.0)$ & 0.252 \\
\hline Diabetes mellitus & $2609(21.5)$ & $233(19.1)$ & $2376(21.8)$ & 0.029 \\
\hline Hypertension & 7748 (63.9) & $763(62.5)$ & $6985(64.1)$ & 0.283 \\
\hline Hyperlipidemia & $1368(11.3)$ & $96(7.9)$ & $1272(11.7)$ & $<0.001$ \\
\hline CHD & $1759(14.5)$ & $246(20.2)$ & $1513(13.9)$ & $<0.001$ \\
\hline Atrial fibrillation & $897(7.4)$ & $140(11.5)$ & $757(6.9)$ & $<0.001$ \\
\hline Pneumonia & 1411 (11.6) & $228(18.7)$ & $1183(10.9)$ & $<0.001$ \\
\hline Living status, no. (\%) & & & & $<0.001$ \\
\hline Living alone & $428(3.6)$ & $245(20.3)$ & $183(1.7)$ & \\
\hline Living with family & $11,563(96.0)$ & $936(77.7)$ & $10,627(98.1)$ & \\
\hline Nursing home & $50(0.4)$ & $24(2.0)$ & $26(0.2)$ & \\
\hline Baseline NIHSS group, no. (\%) & & & & $<0.001$ \\
\hline$<4$ & $10,028(82.8)$ & 959 (78.7) & $9069(83.2)$ & \\
\hline $5-14$ & 1664 (13.7) & $184(15.1)$ & $1480(13.6)$ & \\
\hline$>14$ & $422(3.5)$ & $75(6.2)$ & $347(3.2)$ & \\
\hline \multicolumn{5}{|l|}{ Socioeconomic status } \\
\hline Educational level, no. (\%) & & & & $<0.001$ \\
\hline ESB & $5535(45.7)$ & $788(64.6)$ & 4747 (43.6) & \\
\hline MS & $3138(25.9)$ & $216(17.7)$ & $2922(26.8)$ & \\
\hline HSA & $3445(28.4)$ & $216(17.7)$ & 3229 (29.6) & \\
\hline Occupational class, no. (\%) & & & & $<0.001$ \\
\hline Non-manual workers & $1598(13.2)$ & $91(7.5)$ & $1507(13.8)$ & \\
\hline Manual workers & $3282(27.1)$ & $232(19.0)$ & $3050(28.0)$ & \\
\hline No job & $1278(10.5)$ & $230(18.9)$ & $1048(9.6)$ & \\
\hline Retired & $5146(42.5)$ & $598(49.0)$ & $4548(41.7)$ & \\
\hline Unknown & $814(6.7)$ & $69(5.7)$ & $745(6.8)$ & \\
\hline Personal income, RMB/mon, no. (\%) & & & & 0.092 \\
\hline$\leq 1000$ & $4165(34.4)$ & $447(36.6)$ & $3718(34.1)$ & \\
\hline $1000-3000$ & $4081(33.7)$ & $420(34.4)$ & $3661(33.6)$ & \\
\hline $300-5000$ & $649(5.4)$ & $50(4.1)$ & $599(5.5)$ & \\
\hline$>5000$ & $153(1.3)$ & $13(1.1)$ & $140(1.3)$ & \\
\hline Unknown & $3070(25.3)$ & $290(23.8)$ & $2780(25.5)$ & \\
\hline
\end{tabular}

$I Q R$ inter-quartile range, $B M I$ body mass index, ESB elementary school or below, $M S$ middle school, HSA high school or above, BHIS Basic Health Insurance Scheme for urban and governmental, NCMS New Cooperative Medical System, CHD coronary heart disease, NIHSS National Institutes of Health Stroke Scale 
Fig. 1 Proportions of 1-year stroke outcomes according to marital status
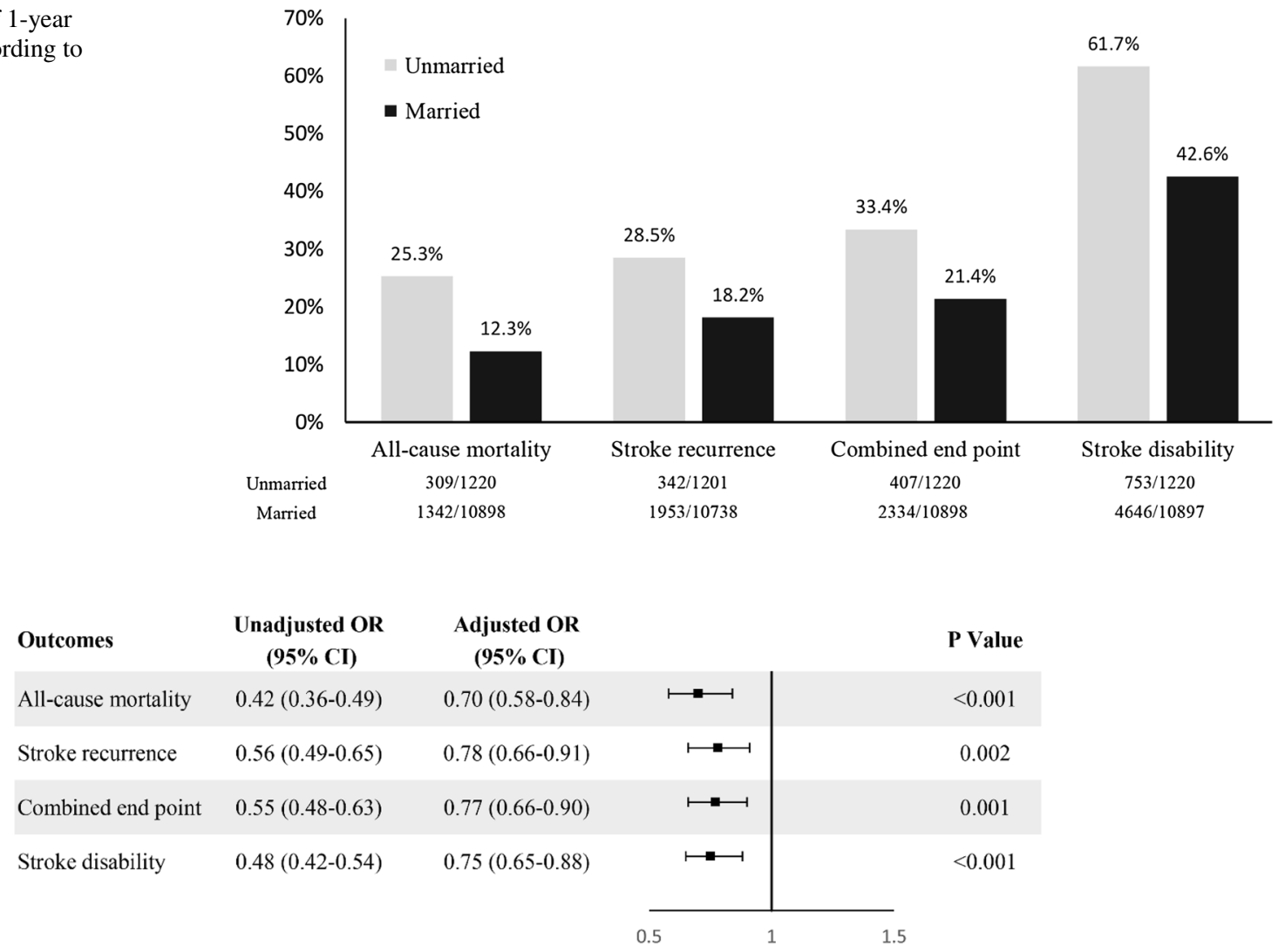

Fig. 2 Odds ratios of being married versus being unmarried for allcause mortality, stroke recurrence, combined end point, and stroke disability. $O R$ odds ratio, $C I$ confidence interval. Covariates included age, sex, region, types of health insurance, history of stroke, hypertension, diabetes, dyslipidaemia, atrial fibrillation, coronary heart

only $33 \%$ subjects were married. In addition, patients who had pre-stroke disability and received help at home were more likely classified into social isolation, which might lead to bias of misclassification.

Studies about the association between living arrangements and stroke outcomes are controversial. The previous studies have showed that patients living alone were more likely to die after stroke episode [12]. However, a recent study found patients living alone had delayed hospital arrival and less thrombolytic therapy, but no significant differences in mortality or readmissions by living status [13]. In the current study, association between living arrangement and stroke outcomes was analyzed only among unmarried patients, since living alone nearly did not happen among married patients. We did not find the association between living status and stroke outcomes in unmarried patients. The plausible explanation would be that living-alone patients might crossover to live with their families for their daily care after stroke, especially patients with moderate-to-severe stroke. The further analysis for this was not made, because we did not collect data on living status during follow-up period. disease, pneumonia, current or previous smoking, moderate or heavy alcohol, body mass index (BMI) at admission, baseline NIHSS, living arrangements, and socioeconomic status (educational level, occupational class, and personal income level)

In our study, stratification analysis showed that effects of marriage on stroke outcomes were significant in patients with middle-school education but not in those with high-or-above education. Furthermore, its interaction for all-cause mortality was significant. We speculated that patient with high school or above education were more likely to have better social support resources, which might partly account for or weaken the protection effect of marriage. In addition, our study found that the association between marital status and stroke outcomes was similar, regardless of whether occupational class and personal income level were the adjustment factors. The previous studies on education related to stroke outcomes were insufficient. A Swedish study found high education and income were associated with a reduced risk of stroke recurrence [14]. A Danish study found education had only a modest effect on survival after stroke episode and only in patients aged $<65$ years [15]. Future studies would be necessary to assess the association between education and marriage and their effects on outcomes among patients with stroke.

At present, the prevailing argument about the protective effect of marriage is that marriage might provide more 


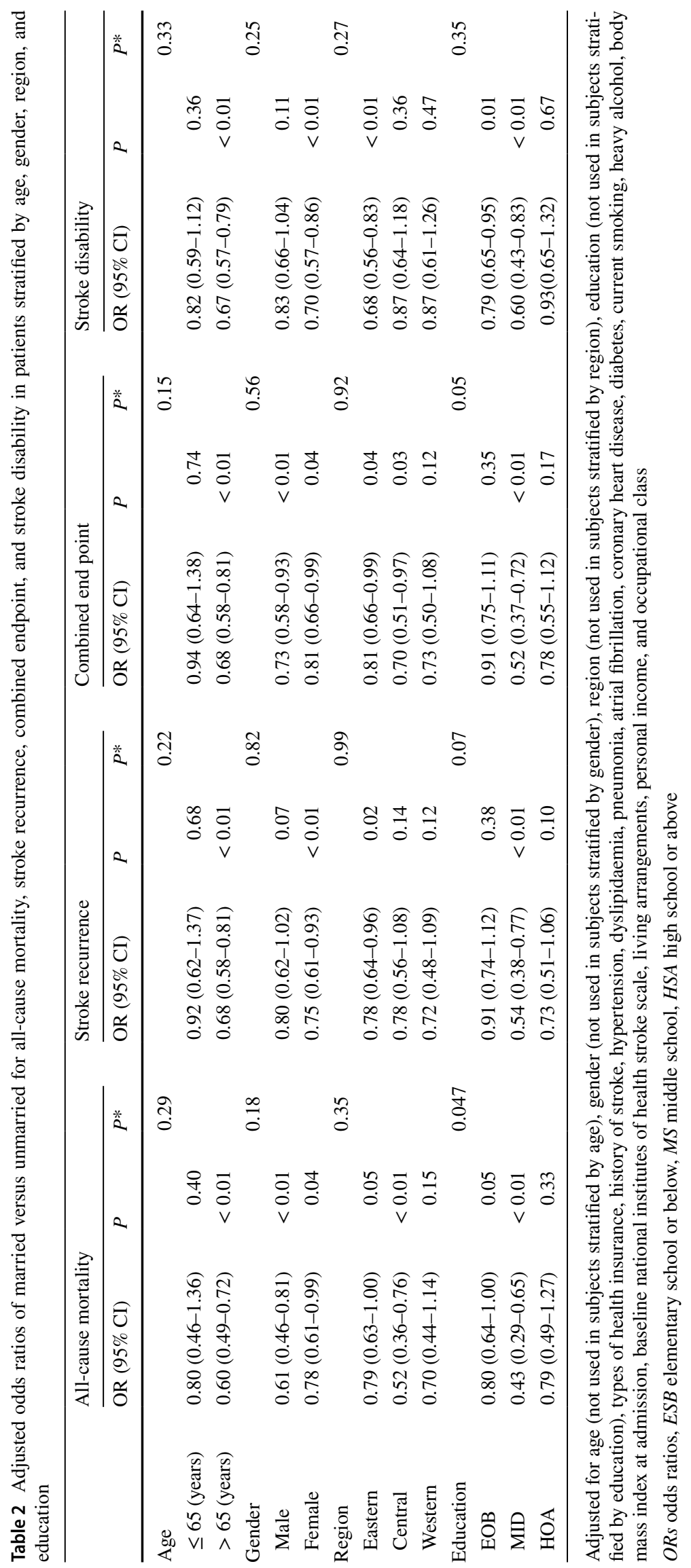


stable behavioral and psychosocial resources to prevent and treat illness [13]. In addition, possible pathophysiological mechanisms related to stress of unmarried have also been reported $[16,17]$. Although marital status is not amenable to medical intervention or treatment, understanding the mechanism could help us to identify possible interventions to reduce these risks, and that is the important area for our future research.

Our study also has limitations. First, although we have adjusted many covariates known to be associated with adverse outcomes, there may be residual confounding which potentially influence our results. In addition, we could not evaluate the level of anxiety or depression in unmarried patients in the study, which might have an interaction for the association. Second, the study was performed based on the Chinese stroke patients, so it would be not generalizable to other races or ethics in which cultural discrepancy in marriage might exist. Finally, this was an observational study, so the results did not imply causation of marriage with stroke outcomes.

In conclusion, marriage was independently associated with post-stroke outcomes in patients with acute ischemic stroke, especially in those with middle-school education. Identifying the association could help us to provide the patients with risk awareness related to marital status.

Funding Funding for this study was provided by the National Key R\&D Program of China (2017YFC1310900 and 2017YFC1310901) and the Ministry of Science and Technology of the People's Republic of China (2012ZX09303 and 2011BAI08B02).

\section{Compliance with ethical standards}

Conflicts of interest The authors declare that they have no conflict of interest.

Ethical standard This study has been approved by our Insititution's Ethics Committee and has therefore been performed in accordance with the ethical standards laid down in the 1964 Declaration of Helsinki and its later amendments.

Open Access This article is distributed under the terms of the Creative Commons Attribution 4.0 International License (http://creativeco mmons.org/licenses/by/4.0/), which permits unrestricted use, distribution, and reproduction in any medium, provided you give appropriate credit to the original author(s) and the source, provide a link to the Creative Commons license, and indicate if changes were made.

\section{References}

1. Feigin VL, Forouzanfar MH, Krishnamurthi R et al (2014) Global and regional burden of stroke during 1990-2010: findings from the Global Burden of Disease Study 2010. Lancet 383(9913):245-254

2. DALYs GBD, Collaborators H, Murray CJ et al (2015) Global, regional, and national disability-adjusted life years (DALYs) for
306 diseases and injuries and healthy life expectancy (HALE) for 188 countries, 1990-2013: quantifying the epidemiological transition. Lancet 386(10009):2145-2191. https://doi.org/10.1016/ S0140-6736(15)61340-X

3. Liu L, Wang D, Wong KS, Wang Y (2011) Stroke and stroke care in China: huge burden, significant workload, and a national priority. Stroke 42(12):3651-3654. https://doi.org/10.1161/STROK EAHA.111.635755

4. Dupre ME, George LK, Liu G, Peterson ED (2015) Association between divorce and risks for acute myocardial infarction. Circ Cardiovasc Qual Outcomes 8(3):244-251. https://doi.org/10.1161/ CIRCOUTCOMES.114.001291

5. Floud S, Balkwill A, Canoy D et al (2014) Marital status and ischemic heart disease incidence and mortality in women: a large prospective study. BMC Med 12:42. https://doi. org/10.1186/1741-7015-12-42

6. Manzoli L, Villari P, Pirone GM, Boccia A (2007) Marital status and mortality in the elderly: a systematic review and meta-analysis. Soc Sci Med 64(1):77-94. https://doi.org/10.1016/j.socsc imed.2006.08.031

7. Dupre ME, Lopes RD (2016) Marital history and survival after stroke. J Am Heart Assoc 5(12):e004647. https://doi.org/10.1161/ JAHA.116.004647

8. Venna VR, McCullough LD (2015) Role of social factors on cell death, cerebral plasticity and recovery after stroke. Metab Brain Dis 30(2):497-506. https://doi.org/10.1007/s11011-014-9544-1

9. Wang Y, Cui L, Ji X et al (2011) The China National Stroke Registry for patients with acute cerebrovascular events: design, rationale, and baseline patient characteristics. Int J Stroke 6(4):355-361. https://doi.org/10.1111/j.1747-4949.2011.00584.x

10. Pan Y, Chen R, Li Z et al (2016) Socioeconomic status and the quality of acute stroke care: the china national stroke registry. Stroke 47(11):2836-2842. https://doi.org/10.1161/STROK EAHA.116.013292

11. Boden-Albala B, Litwak E, Elkind MS, Rundek T, Sacco RL (2005) Social isolation and outcomes post stroke. Neurology 64(11):1888-1892. https://doi.org/10.1212/01.WNL.0000163510 .79351.AF

12. Redfors $P$, Isaksen $D$, Lappas $G$ et al (2016) Living alone predicts mortality in patients with ischemic stroke before 70 years of age: a long-term prospective follow-up study. BMC Neurol 16:80. https ://doi.org/10.1186/s12883-016-0599-y

13. Reeves MJ, Prager M, Fang J, Stamplecoski M, Kapral MK (2014) Impact of living alone on the care and outcomes of patients with acute stroke. Stroke 45(10):3083-3085. https://doi.org/10.1161/ STROKEAHA.114.006520

14. Pennlert J, Asplund K, Glader EL, Norrving B, Eriksson M (2017) Socioeconomic status and the risk of stroke recurrence: persisting gaps observed in a nationwide Swedish Study 2001 to 2012. Stroke 48(6):1518-1523. https://doi.org/10.1161/STROK EAHA.116.015643

15. Andersen KK, Dalton SO, Steding-Jessen M, Olsen TS (2014) Socioeconomic position and survival after stroke in Denmark 2003 to 2012: nationwide hospital-based study. Stroke 45(12):35563560. https://doi.org/10.1161/STROKEAHA.114.007046

16. Hafner S, Baumert J, Emeny RT et al (2012) To live alone and to be depressed, an alarming combination for the renin-angiotensin-aldosterone-system (RAAS). Psychoneuroendocrinology 37(2):230-237. https://doi.org/10.1016/j.psyneuen.2011.06.007

17. Molloy GJ, Stamatakis E, Randall G, Hamer M (2009) Marital status, gender and cardiovascular mortality: behavioural, psychological distress and metabolic explanations. Soc Sci Med 69(2):223-228. https://doi.org/10.1016/j.socscimed.2009.05.010 